YAN ZHU and FREDERICK K. S. LEUNG

\title{
MOTIVATION AND ACHIEVEMENT: IS THERE AN EAST ASIAN MODEL?
}

Received: 25 February 2010; Accepted: 27 October 2010

\begin{abstract}
The importance of motivation in learning has been widely recognized. However, due to its multidimensional and complex nature, it appears difficult to synthesize research findings on motivation across studies. Heated debates about the effects of intrinsic and extrinsic motivation on learning and their interaction have been going on since the terms started to be used. Moreover, cultural difference acts as another crucial factor in the field. Using the Trends in International Mathematics and Science Studies 2003 eighth grade mathematics data, this study scrutinized the relationship between pleasure-oriented (intrinsic-related) and productivity-oriented (extrinsic-related) motivation and how they collectively affect students' academic performance in East Asian education systems compared with those from Australia, England, The Netherlands, and the USA. The study found that both types of motivation contributed to East Asian students' mathematics achievement in an additive fashion, whereas extrinsic-related motivation appeared to have a detrimental effect on their Western counterparts' learning. Possible reasons were explored from a cultural perspective.
\end{abstract}

KEY WORDS: East Asia, extrinsic motivation, intrinsic motivation, mathematics achievement, pleasure-oriented motivation, productivity-oriented motivation, TIMSS

The importance of motivation in learning has been widely recognized. It is believed that motivation provides the primary impetus to initiate learning and later the driving force to sustain the learning (e.g. Guilloteaux \& Dörnyei, 2008; Wang, 2008). Some further claimed that motivation is central to student learning (e.g. Mankin, Boone, Flores, \& Willyard, 2004). It is believed that motivation is among the most powerful determinants of students' success or failure in school (e.g. Ryan \& Connell, 1989; Sternberg \& Wagner, 1994). Means, Jonassen \& Dwyer (1997) cited studies showing that motivation accounted for $16 \%$ to $38 \%$ of the variations in overall student achievement. Consistently, in mathematics education, motivation has also been regarded as one of the most important issues (Walker \& Guzdial, 1999). Hannula (2006) further highlighted that in order to understand students' various behaviors in mathematics classrooms, including those unexpected, it was important to increase our understanding of what motivation was and how it was regulated.

International Journal of Science and Mathematics Education (2011) 9: 1189-1212

(C) The Author(s) (2010). This article is published with open access at Springerlink.com 
Nevertheless, it appears difficult to synthesize research findings on motivation across studies due to the inconsistency in the ways in which motivation has been defined and measured (e.g. Early, 2006; Harlen \& Deakin, 2002). For instance, a study by Kleinginna \& Kleinginna (1981) found a total of 102 statements defining or criticizing the concept of motivation from a variety of sources, and these statements were further classified into nine categories based on the phenomena or theoretical issues emphasized with a tenth category containing the skeptical statements. The inconsistency on the definition of motivation, as suggested by Holden (1990), is related to its unobservable nature. In particular, one's motivation can be judged only through one's behavior and surroundings in which one is active. In psychology, according to Geen (1995), motivation addresses the initiation, intensity, and persistence of human behavior; in other words, motivation could be viewed as the power that helps a person initiate a persistent behavior with a certain degree of intensity in order to achieve a long-term goal (also see Li, Davis \& Lomax, 2008). Similarly, Franken (1994) suggested three dimensions of human behavior to exemplify the notion of motivation, which are arousal, direction, and persistence. There are also researchers who proposed other components in behavior related to motivation, such as continuity (Hebb, 1955), energization (Deci \& Ryan, 1985), and performance (Simms, 1998).

To elaborate the complex nature of motivation, a vast number of motivational theories have been developed since motivation began to be studied as a discipline separate from learning in the 1930s. Early motivation researchers primarily focused on the factors which stimulated behavior. Many theories at that time were established based on the idea of homeostatic balance. Clark Hull's drive reduction theory was one of such theories, which interprets motivation as the desire to reduce drives to a state of neutrality (Model, 2005). With the emergence of cognitive science in the $1950 \mathrm{~s}$, motivation researchers shifted their interests to the direction of behavior (Ngaosuvan, 2004). One important cognitive motivation theory is Victor Vroom's expectancy-value theory that views motivation as a product of the perceived probability of success and the value of that success (Demmrich, 2005). From the 1970s, voluntarism which heavily emphasizes on the self has started to dominate motivational research. Deci and Ryan's self-determination theory is such a representative and influential one developed in this context. The theory begins with the assumption that people are active organisms with inborn tendencies toward psychological growth and development. It suggests that humans have three innate needs: competence, autonomy, and relatedness. 
Regarding the history of motivational studies, Weiner (1990) commented that the field has demonstrated great vigor and movement, while there were still many uncharted areas in front of motivational researchers.

It is clear that no single theory or model can account for all aspects of human motivation. However, each of the major approaches makes contributions to our understanding of motivation. Compared to the variety of motivational theories, it seems that researchers have more consensuses on the classification of motivation types. There are in essence two types of motivation, namely intrinsic motivation and extrinsic motivation. The former refers to motivation that comes from inside an individual, while the source for the latter comes from others. Vallerand, Pelletier, Blais, Briere, Senecal \& Vallieres (1992) further differentiated intrinsic motivation into three more specific types, that is, to know (i.e. one engages in an activity for the pleasure that one experiences while learning or trying to something new), to accomplish things (i.e. one engages in an activity for the satisfaction that is derived from achieving or creating something), and to experience stimulation (i.e. one engages in an activity for the stimulating sensations one receives from participating). In terms of sources of intrinsic motivation, Lindenberg (2001, 2003) introduced distinction between enjoyment-based "hedonic" and obligation/community-based "normative" intrinsic motivation. According to Lindenberg (2001), the former is about achieving physical and social wellbeing and allowing improvement in one's condition, while the latter is about the wish to act appropriately in certain contexts that is acquired through socialization. Similarly, extrinsic motivation can also be further classified. For instance, Deci \& Ryan (1985) suggested four different types of extrinsic motivation ordered along a continuum of autonomy, namely external, introjected, identified, and integrated regulation. In particular, external regulation refers to behaviors controlled by external rewards or constraints; introjected regulation refers to behaviors out of avoiding pressure, guilty, or obtaining self-esteem; identified regulation refers to behaviors that are judged important and perceived as chosen by the individual; and integrated regulation refers to behaviors that correspond to the sense of self.

Many believed that learning behavior is a product of either intrinsic or extrinsic motivation (Hayamizu, 2002). However, regarding the effects of the two types of motivations on learning and their interaction, heated debates have been going on since these motivational terms started to be used. It is typically deemed that intrinsic motivation tends to be deeper and more powerful than the extrinsic one and its corresponding effect also tends to last longer. The argument about the superiority of intrinsic 
motivation usually relates to its nature, that is, it originates from within the individual and is independent of external influences. In contrast, researchers viewed the temporariness as one serious drawback of extrinsic motivation, that is to say, such motivation would disappear as soon as the reward or punishment was withdrew. In his book Published by Rewards, Kohn (1993) listed another two important drawbacks of extrinsic motivation, which were getting diminishing returns (i.e. the motivation would slowly drop off if the reward or punishment maintains at the same levels) and hurting intrinsic motivation (i.e. the reward or punishment would remove people's innate desire to do things on their own).

As regards the undermining effect of extrinsic motivation, Ryan \& Deci (1999) interpreted it in terms of the reward shifting people from internal to external perceived locus of causality. Similarly, Amabile (1983) argued that extrinsic motives could cause people to divide their attention between extrinsic goals and the task at hand (also see Sternberg, 1999). Some further suggested that the existence of extrinsic motivation is detrimental toward other aspects of learning, such as creativity (e.g. Amabile, Schatzel, Moneta \& Kramer, 2004; Deci \& Ryan, 1985). Consequently, educators have been suggested to use effective strategies to enhance students' intrinsic motivation while avoiding the use of extrinsic motivation in school as much as possible (Tang, 2008).

However, there are some researchers suggesting that intrinsic and extrinsic motivations may not necessarily be polar opposites (e.g. Lepper, Corpus \& Lyengar, 2005). For instance, in Bateman \& Crant's (2003) study with more than 800 undergraduate alumni from a large private university, extrinsic rewards and values often demonstrated significant positive main effects, rather than undermining effects, on intrinsic outcomes, particularly when intrinsic needs were low (also see Brophy, 2004). A similar statement has been made by Cameron (2001) that extrinsic motivation could be helpful when intrinsic motivation is low. In particular, she argued that people often do things which are not intrinsically motivated so that they may simply lose interest in doing them without external rewards.

The above antagonistic opinions indicate that the interaction between the two types of motivations is a complex process which could be affected by many relevant factors. In fact, researchers found that different types of extrinsic rewards could play different roles on intrinsic motivation. In particular, while expected, tangible, and task-contingent rewards typically undermine intrinsic motivation, unexpected rewards, verbal rewards and positive feedback, and task-noncontingent rewards promote intrinsic motivation (Evertson \& Weinstein 2006). Lepper, 
Keavney \& Drake, (1996) summarized that extrinsic rewards indicating success and/or increasing feeling of competence could strengthen intrinsic motivation with those providing constraints or social controls having reducing effects on intrinsic motivation. Similarly, Deci (1980) identified the two opposite functions as two elements of extrinsic reward: the informational aspect and the controlling aspect. According to him, the informational aspect enhances intrinsic motivation and the controlling aspect leads to a decrease in intrinsic motivation (also see Bolt \& Myers 1984).

Baker, Dreher \& Guthrie (2000) suggested that maintaining a balanced perspective on this debate perhaps is the best way to resolve the problem. Lepper \& Henderlong (2000) also pointed out that people can be high in both or low in both motivations, not just high in one and low in the other. In brief, the two can and do coexist with one another, and furthermore, they could complementarily influence behavior.

Besides the multidimensional and complex nature of motivation, culture difference is another crucial factor in the field. For instance, $\mathrm{Ng}$ (2003) attributed the motivation differences related to the Asian societies to their collectivist cultures and the associated schooling practices. In particular, parents and school teachers in Asia generally believe that "learning cannot be separated from achievement" and "learning and achievement are social obligations". $\mathrm{Ng}$ argued that such cultural influences resulted in the continuous emphasis on performance goals; correspondingly, learning for personal interest, enjoyment, and improvement was less treasured. Similarly, Markus \& Kitayama (1991) argued that Asian individuals tended to derive their motivations from what benefits others and a group as a whole, while people in Western cultures tended to have self-benefiting motivations. Responsibility to the family and meeting family expectations are salient in self processes from Asian cultural settings (Walker \& Debus, 2002). Pertaining to the subject of mathematics, Leung (2001) maintained that educators in the West place a high value on intrinsic motivation in learning but regard extrinsic motivation (such as pressure from examination) as a harmful source, which, however, is acceptable in the East Asian countries. In this sense, without taking this vital factor into account, one could hardly understand why East Asian students have consistently outperformed academically their counterparts around the world in international comparison studies but always reported their motivation at a lower level (e.g. Programme for International Student Assessment, Trends in International Mathematics and Science Studies (TIMSS)).

Using the TIMSS 2003 mathematics data, this study scrutinized the relationship between intrinsic and extrinsic motivation and how the two 
collectively affect students' academic outcomes in East Asian education systems. To identify the possible characters peculiar to East Asian culture, data from four Western systems (i.e. Australia, England, The Netherlands, and the USA) were included in the analysis as a reference. These systems are included in the analysis not only because they are all typically Western ones but also because the curricula in these countries have great impact on the East Asian mathematics curricula.

\section{Research Methods}

\section{Students}

The data for this study were taken from the TIMSS 2003 eighth grade mathematics study. About 49 education systems around the world participated in the TIMSS 2003, and nine of them were included in the current study: Five were from East Asia (i.e. Hong Kong SAR, Japan, Republic of Korea, Singapore, and Chinese Taipei) and the other four were Western countries including Australia, England, The Netherlands, and the USA. In all, there were 4,972 students from the Hong Kong sample, 4,856 from Japan, 5,309 from Korea, 6,018 from Singapore, 5,379 from Chinese Taipei, 4,791 from Australia, 2,830 from England, 3,065 from The Netherlands, and 8,912 from the USA.

\section{Measures}

The TIMSS 2003 instrument consists of tests of students' achievements in mathematics and science, along with background questionnaires for students, teachers, and principals. The background information allows cross-national comparison of educational contexts so as to investigate the differences existed in students' academic performance. The focus of this study is about students' motivational factors, and the relevant information was solely taken from the student background questionnaire. Though TIMSS was not specifically designed to address motivation issues, pertaining to the subject of mathematics, there were seven relevant items asking about students' enjoyment and value in learning the subject. In particular, one item measured whether students experienced pleasure from learning mathematics (BSBMTENJ), two related to students' preference for mathematics in future study (BSBMTMOR) and job (BSBMAJOB), and the other four were about students' perception of the usefulness of mathematics (BSBMAHDL, BSBMAOSS, BSBMAUNI, and BSBMAGET; a list of all the items is included in the "Appendix"). In the TIMSS 
questionnaire, students were asked to rate their motivation level on a fourpoint Likert scale ranging from 1 (strongly agree) to 4 (strongly disagree).

In the TIMSS mathematics assessment, each student was randomly assigned with one out of 12 booklets containing only a part of the assessment item pool. The raw test scores thus are not comparable across students. To resolve the problem, TIMSS used multiple imputation approach to generate proficiency scores in mathematics for all students, which represent an estimation of each student's performance if he/she had actually answered all items (Gonzalez, Galia \& Li, 2004). As a result, five plausible values were produced for each student, and all of them were used in the current study to calculate parameter estimates.

\section{Data Processing and Analysis}

Principal component factor analysis ${ }^{1}$ was first performed to determine whether the seven motivational items could be explained by a smaller number of underlying factors. Promax rotation was used to decide whether the factor structures were correlated or independent. To assess the internal consistency of the factors, Cronbach's alpha coefficients were calculated. All these analyses were performed on the weighted data (i.e. TOTWGT as the weighting variable) so as to ensure that the various subgroups that constitute each education system's sample are properly and proportionally represented in the computation of population estimated for that system (Foy \& Joncas, 2004). Based on the factor model, composite scores were created for the followed up analyses.

For each education system, descriptive analysis on individual motivation items and motivational factors was first carried out. Possible cross-system differences were investigated via a series of $t$ tests. Effect sizes of the differences were reported when the discrepancies were found at a statistically significant level. After this, the differences between different motivational factors within and across systems were examined. Lastly, in order to determine the effects of the different motivational factors on students' achievement results, correlations among those factors and mathematics achievement followed by regression analyses were conducted. To facilitate the interpretation of the analysis results, the scales were reversed in an ascending order in correlation analysis and regression analysis. All the analyses were conducted using SPSS and AM software, a statistical software package used to examine large data sets with complex sampling designs such as the TIMSS (Cohen, 2005). 


\section{Results AND Discussion}

\section{Factor and Item Analysis}

In order to determine the factor structure of the motivation scale in the TIMSS, the seven relevant items were analyzed using principal component factor analysis. Kaiser-Meyer-Olkin measure of sampling adequacy of 0.82 and a significant Bartlett's test of sphericity first suggested that the data set was well suited for the analysis. As a result, two factors emerged with eigenvalues over one (explaining $48.88 \%$ and $15.13 \%$ of the variance), and all the items had primary loadings over 0.45 with one item having a cross-loading above 0.40 (BSBMAJOB). The result is somewhat expected as both BSBMAJOB and BSBMAGET assess students' perceptions about their future job, and furthermore, the two items had a moderate correlation $(r=0.56)$.

In order to identify which underlying attributes the two job-related items actually belonged to, two follow-up factor analyses were carried out; in particular, BSBMAGET was removed in the first analysis, and BSBMAJOB was excluded in the second one. The results are presented in Table 1, which illustrated a clearer factor structure of the seven motivation items. It can be seen that BSBMAJOB together with BSBMTMOR and BSBMTENJ formed factor 1, which was related to students' enjoyment and preference for mathematics, and it was thus named pleasure-oriented motivation (PLM), similar to Lindenberg's (2001) enjoyment-based intrinsic motivation. The other four items, including BSBMAGET, were under factor 2, and all of the items were

TABLE 1

Rotated factor loading for the motivational items

\begin{tabular}{|c|c|c|c|c|c|}
\hline \multicolumn{3}{|c|}{ Factor analysis without BSBMAGET } & \multicolumn{3}{|c|}{ Factor analysis without BSBMAJOB } \\
\hline$K M O=0.80$ & Factor 1 & Factor 2 & $K M O=0.77$ & Factor 1 & Factor 2 \\
\hline BSBMTMOR & 0.91 & -0.09 & BSBMTMOR & 0.90 & -0.02 \\
\hline BSBMTENJ & 0.90 & -0.05 & BSBMTENJ & 0.89 & 0.00 \\
\hline BSBMAHDL & 0.12 & 0.72 & BSBMAHDL & 0.20 & 0.64 \\
\hline BSBMAOSS & -0.04 & 0.84 & BSBMAOSS & 0.07 & 0.72 \\
\hline BSBMAUNI & -0.08 & 0.81 & BSBMAUNI & -0.14 & 0.86 \\
\hline BSBMAJOB & 0.58 & 0.29 & BSBMAGET & -0.03 & 0.79 \\
\hline$\%$ of variance & 49.84 & 16.69 & $\%$ of variance & 17.50 & 48.83 \\
\hline Correlation & 0.49 & & Correlation & 0.44 & \\
\hline
\end{tabular}


about students' perceptions of the usefulness of learning mathematics. Correspondingly, factor 2 was named productivity-oriented motivation (PRM) in this study (i.e. learning for utilitarian purposes), similar to Deci \& Ryan's (1985) identified regulation in extrinsic motivation. The two component correlation matrices showed a moderate inter-item correlation between the two factors, which indicates the appropriateness of using promax rotation in the factor analysis. The Cronbach's alpha values for the two factors were 0.77 and 0.76 , respectively, and this further signifies adequate internal consistency.

Table 2 presents the weighted descriptive statistics of students' responses to individual motivational items as well as the composite scores $^{2}$ on the two types of motivation (i.e. PLM and PRM) across the nine educational systems. Due to the fact that the Likert scale in the questionnaire is in a descending order of agreement, a higher score indicates a lower level of motivation. The results showed that students from the USA had the highest level of productivity-oriented motivation, which is significantly higher than that of the students from all the East Asian systems as well as the other three Western ones. In particular, the magnitudes of the differences between the USA and the two Commonwealth systems are about small (USA-Australia: $d=0.21$; USA-England: $d=0.45$ ) and those with the East Asian systems are about medium (USA-Hong Kong: $d=0.68$ ) or large (USA-Japan: $d=1.21$, USAKorea: $d=1.00$, USA-Taiwan: $d=0.95$ ) except trivial with Singapore $(d=0.11)$. Interestingly, the students from The Netherlands reported a similar level of PRM as those from Korea, Chinese Taipei, and Japan; either no significant or trivial differences were observed among the four systems.

For pleasure-oriented motivation, Singaporean students reported the highest level followed by the US students, and the difference between the two systems is about small in terms of effect size $(d=0.37)$. Compared to PRM, the differences in PLM between the USA and the other seven systems are smaller, and the magnitudes are mostly about small (i.e. $d<0.50$ ). Only the students from Korea and The Netherlands showed a slightly lower level of PLM, and their differences from the US peers were about medium size (USA-Korea: $d=0.58$; USA-The Netherlands: $d=0.71$ ).

Within the five East Asia education systems, the students from Singapore and Hong Kong illustrated a higher level of similarity compared to the other three. In fact, the difference in PLM between the two systems is about small in terms of effect size $(d=0.45)$, whereas the corresponding differences between Singapore and the other three East 
Asian systems are about medium (Singapore-Taiwan: $d=0.74$; SingaporeJapan: $d=0.77$ ) or large (Singapore-Korea: $d=0.90$ ). Though the difference in PRM between Singapore and Hong Kong is comparatively larger than that in PLM $(d=0.64)$, the discrepancy between Singapore and the other three systems is even greater (Singapore-Korea: $d=1.02$; Singapore-Taiwan: $d=1.04$; Singapore-Japan: $d=1.21$ ). Given the fact that the two systems have many similarities, especially in their historical background and status quo, the results seem understandable and also somehow expected. While both systems receive deep impact of the Western cultural influences, traditional eastern cultures are still thriving. Such a fusion of the two seemingly conflicting types of culture actually makes the two systems unique in the region in many other aspects as well.

Among the four PRM items, the item on the importance of mathematics for getting a desired job (BSBMAGET) received least agreement, which occurred in all the nine educational systems. It may be related to the fact that the participating students were at the lower secondary level (i.e. grade 8) so that getting a job was a farther target than entering a preferred university. As revealed in Table 2, students from five out of the nine systems had the highest agreement on the relevant item (i.e. BSBMAUNI). Consistently, students from the five East Asian systems and the USA also had the least agreement on the statement about job involving mathematics among the three PLM items (i.e. BSBMAJOB). The Dutch students were the only group who had a higher agreement on this item than the other PLM items. In addition, it is interesting that the students from both Hong Kong and Chinese Taipei had more agreement on the item about the usefulness of mathematics in their daily life than getting into university, which was also found in the two Commonwealth systems.

Comparing the two types of motivation, the analysis showed that students from all the nine educational systems had significantly higher level of agreement on the statements related to the utilitarian one. The corresponding difference is much greater in the Western systems than the East Asian ones. In particular, except for Korea $(d=1.02)$, the magnitude of the differences in the other four East Asian systems is about medium (Hong Kong: $d=0.77$; Japan: $d=0.75$; Singapore: $d=0.70$; Taiwan: $d=0.71$ ), and the magnitude in all the four Western systems is large (Australia: $d=1.02$; England: $d=1.17$; The Netherlands: $d=1.13$; USA: $d=1.11$ ). Furthermore, the data showed that more than $70 \%$ of the students in all the systems had a higher level of PRM than that of PLM, and the percentages in Korea and the four Western systems were even higher than $83 \%$. 
Relationships Among Pleasure-Oriented Motivation, ProductivityOriented Motivation, and Mathematics Achievement

In the literature, researchers often found that externally oriented motivation correlated to internally oriented motivation in a negative manner. Some researchers even argued that extrinsic motivation had an undermining effect on intrinsic motivation. However, as discussed earlier, extrinsic motivation is a complex process, which comprises various types. Different types of extrinsic motivation could have different relationship with intrinsic motivation. For instance, Lepper et al. (1996) suggested that extrinsic motivation promoting success and competence would enhance intrinsic motivation, but those providing constrains or controls diminish intrinsic motivation. Table 3 clearly reveals a moderate positive correlation between PLM and PRM, which occurs in all the nine educational systems. This finding supports Lepper et al.'s argument as the four PRM items measured in the TIMSS 2003 are about students' perception of utility value of learning mathematics, which could promote one's feeling of success and/or competence rather than giving constraints. According to Deci \& Ryan (1991), though this type of motivation is externally oriented, it is more autonomous or self-determined as with this type of motivation, one experiences a sense of direction and purpose instead of constraints or pressures. Such choices and opportunities for self-direction were found to enhance internally oriented motivation because they bring people a greater feeling of autonomy (e.g. Deci \& Ryan, 1985, 2000).

In most cases, while both types of motivation show positive correlations with students' mathematics achievement, the associations are relatively weak, especially for the Western samples. Between the two motivational indicators, PLM has stronger association with students' achievement than PRM. In Singapore, Australia, England, and the USA, the differences are more than twice. Moreover, in The Netherlands, the two types of motivation even show an opposite correlation with mathematics achievement.

When examining the correlation between students' mathematics achievement and individual PLM items, it is interesting to find that for all the five East Asian systems and Australia, the item about enjoyment (BSBMTENJ) demonstrated the strongest correlation with achievement but weakest in the USA. The corresponding magnitude was about twice (Hong Kong: $r=0.32$, Japan: $r=0.31$; Singapore: $r=0.28$; Australia: $r=0.22$ ) or thrice (Korea: $r=0.40$; Taiwan: $r=0.46$ ) of that for the US sample $(r=0.13)$. Regarding the England and The Netherlands data, the differences were even greater (England: $r=0.10$; The Netherlands: $r=0.04)$. For the England, The Netherlands, and the US data, the item 


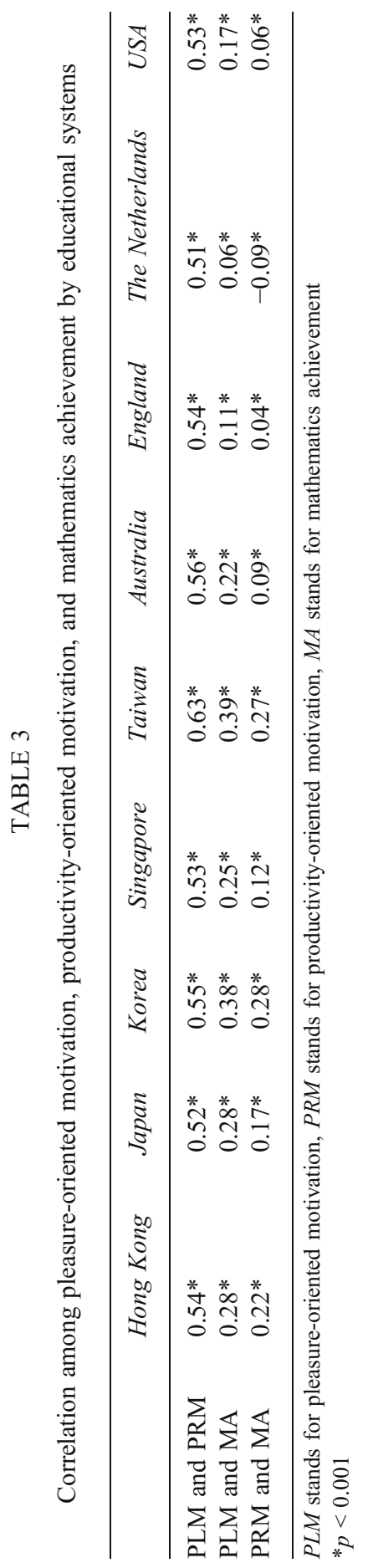


related to having a job involving mathematics showed the highest correlation with academic achievement, though the absolute values were generally lower than those in the East Asian systems and Australia.

Among the four PRM items, the one related to getting into university had the strongest correlation with students' mathematics achievement in all the systems but England and The Netherlands. In particular, the data from Korea $(r=0.31)$ and Chinese Taipei $(r=0.29)$ demonstrated the greatest association. On the other hand, the motivation indicator about the usefulness of mathematics for daily life showed a rather weak association with students' achievement, and in most systems, it was the weakest one. Moreover, in all the Western systems but Australia, there was a negative correlation on this item. Interestingly, while the students from Hong Kong and Chinese Taipei had a higher level of agreement on this motivation indicator than the other three East Asian systems, the corresponding correlation in the two systems was also stronger than that in the rest of the systems.

\section{Influences of Pleasure-Oriented Motivation and Productivity-Oriented Motivation on Mathematics Achievement}

The regression analyses revealed that both motivational factors accounted for significant but a small percentage of the variance in students' mathematics achievement. Among the nine educational systems, the influences from PLM and PRM were most obvious for Chinese Taipei $\left(R^{2}=0.16\right)$ and Korea $\left(R^{2}=0.15\right)$ but least for the USA $\left(R^{2}=0.03\right.$; see Table 4). The general low $R$-squared values indicate that motivation is just one of the many factors which influence students' learning process as well as their learning outcome, and similar arguments were echoed in the literature (e.g. Broussard, 2002; Li \& Pan, 2009; Lim \& Yeon, 2009; Rose, 2007; Wright, 2004). On the other hand, Wittink (1998) suggested that one should focus on the precision of the slope coefficients if the main purpose is to understand the relationship between variables.

In general, both types of motivation demonstrated positive influences on East Asian students' achievement except for Singapore, as revealed in Table 4. For all the four Western systems, when both pleasure-oriented and productivity-oriented motivations were included in the model, the latter showed a negative impact, and in three systems, such an impact reached a statistically significant level. The results seem to suggest that the two types of motivation could and did contribute to students' achievement in an additive fashion in the East Asian educational systems. Though there was a negative influence from PRM found in the Singapore data, its magnitude was not statistically significant. In other words, when both types of motivation 
TABLE 4

Results of regression analysis of two types of motivation on students' mathematics achievement by educational systems

\begin{tabular}{|c|c|c|c|c|c|c|c|}
\hline & \multicolumn{2}{|l|}{ Intercept } & \multicolumn{2}{|l|}{$P L M$} & \multicolumn{2}{|l|}{$P R M$} & \multirow[b]{2}{*}{$R^{2}$} \\
\hline & $\alpha$ & $S E$ & $B$ & $S E$ & $\beta$ & $S E$ & \\
\hline Hong Kong & $672.72 * * *$ & 4.73 & $23.41 * * *$ & 1.82 & $12.95 * * *$ & 2.90 & 0.08 \\
\hline Japan & $671.68 * * *$ & 5.90 & $32.02 * * *$ & 2.02 & $4.79 * * * *$ & 2.51 & 0.08 \\
\hline Korea & $738.99 * * *$ & 5.60 & $40.43 * * *$ & 2.06 & $14.15^{* * *}$ & 2.60 & 0.15 \\
\hline Singapore & $658.02 * * *$ & 4.12 & $25.85 * * *$ & 1.53 & -2.67 & 2.64 & 0.06 \\
\hline Taiwan & $733.60 * * *$ & 6.57 & $48.94 * * *$ & 2.44 & $5.08 * * * *$ & 2.77 & 0.16 \\
\hline Australia & $557.69 * * *$ & 5.52 & $24.57 * * *$ & 2.35 & $-6.41 *$ & 3.09 & 0.05 \\
\hline England & $529.81 * * *$ & 6.88 & $12.86 * * *$ & 2.65 & -3.10 & 3.09 & 0.01 \\
\hline The Netherlands & $535.30 * * *$ & 9.40 & $15.93 * * *$ & 2.91 & $-21.34 * * *$ & 3.01 & 0.03 \\
\hline USA & 540.76 *** & 3.27 & $17.64 * * *$ & 1.26 & $-4.89 * *$ & 1.88 & 0.03 \\
\hline
\end{tabular}

PLM stands for pleasure-oriented motivation, $P R M$ stands for productivity-oriented motivation $* p<0.05 ; * * p<0.01 ; * * * p<0.001 ; * * * * p<0.10$

influenced Singapore students' mathematics achievement, the impact of PRM was trivial. Similar results also applied to the England data. However, in the other three Western systems, PRM appeared to have a detrimental effect, and the influence was particularly strong in The Netherlands.

The results showed that pleasure-oriented motivation played a strongly positive role in students' achievement among all the nine educational systems, which is consistent with what the literature has suggested. The influence appeared the strongest in Chinese Taipei; in particular, a student's mathematics achievement can be enhanced by about 49 points for every increment of one level higher in PLM. With respect to the intercept, the increment is about $6.7 \%$. That is to say, when a student in Chinese Taipei had one level higher in PLM, his/her mathematics achievement could generally be enhanced by $6.7 \%$. Similarly, the improvement in Korea is about 40 points $(5.5 \%)$. The change for England (2.4\%), Netherlands (3.0\%), and US students $(3.3 \%)$ is only about half of what Chinese Taipei students have. Moreover, all these increments are statistically significant at the 0.001 level.

In all the nine educational systems but The Netherlands, the influence of productivity-oriented motivation on students' mathematics achievement is much smaller than that of pleasure-oriented motivation. The data showed that the students from Korea and Hong Kong received greatest benefits from a higher level of PRM for their achievement. In particular, the students from the two systems could achieve nearly $2 \%$ more of score 
points with one level higher in PRM. In contrast, students from The Netherlands achieved about $4 \%$ less of mathematics score points and such a decline was significant at the 0.001 level. In both Australia and the USA, the decrement was around $1 \%$, which also reached a significant level (see Table 4). In sum, pleasure-oriented motivation (as one dimension of intrinsic motivation) was a far stronger motivator than productivity-oriented motivation (as one dimension of extrinsic motivation) in affecting students' mathematics achievement. In East Asian educational systems, both types of motivation seemed to function synergistically and their combination tended to produce a higher level of overall motivation, which is not the case in the west.

\section{CONCLUSIONS AND IMPLiCATIONS}

Using the TIMSS 2003 data, this study examined students' pleasure-oriented and productivity-oriented motivation level pertaining to mathematics learning and the influence of the two types of motivation on students' performance in mathematics assessment at the eighth grade level in five East Asian educational systems and four Western ones. It was found that the students from all the nine systems had significantly higher level of agreement on the externally oriented motivation than the internally oriented one. The magnitude of the differences between the two types of motivation was generally medium in East Asian systems and large in the west. It suggests that the students valued the usefulness of mathematics more than enjoying its learning as an inner force. This result is not a surprise and many researchers have consistently reported that students' enjoyment level in studying mathematics was generally low at the secondary level. For instance, Macnad \& Payne's (2003) survey asked all students in Scotland completing teacher education courses for teaching in primary schools (i.e. in the last year of the 4-year Bachelor of Education (BEd) course or in the 1-year Post-graduate Certificate of Education (Primary) course) together with those in the first year of the BEd course to compare their feelings about mathematics when they were at the primary, secondary, and tertiary levels. It was found that across the three school levels, these student teachers felt that mathematics at the secondary level was least interesting and fun. Comparatively, the student teachers' perceptions about the usefulness of mathematics had not much difference between the primary and secondary levels and more agreement was revealed at the tertiary level.

In general, the US students showed higher motivation level than East Asian students with the only exception of Singapore. Hong Kong 
students' motivation level is more similar to those from the two Commonwealth systems. The other three East Asian systems are more alike with each other. The distinctive blend of East and West could be one important factor which makes Singapore and Hong Kong maintain the Eastern cultural identity while demonstrating some Western characters.

The study showed that the two types of motivation have a moderate positive association. As discussed earlier, the items included in the TIMSS 2003 related to extrinsic motivation are about students' perceptions of the usefulness of mathematics. It is close to Ryan and Deci's so-called identified regulation, and they argued that this dimension of extrinsic motivation is nearer to intrinsic motivation in the autonomy continuum. The positive correlation suggests that when students appreciate the value of mathematics, such a motivation as an external stimulator could enhance the overall learning motivation level. In fact, such a pattern was found in all the nine educational systems.

It was revealed that in eight out of the nine education systems, both pleasure-oriented and productivity-oriented motivations are associated with students' mathematics test scores in a positive manner, though the relevant magnitudes are small in general. Comparatively, the correlation with PLM is much stronger than that with PRM. Many research studies have consistently found that internally oriented motivation is more powerful and effective than externally oriented one, especially in long-period learning. Deci (1980) argued that people desired to be the causal agents of their own behaviors rather than being controlled by the environment. In other words, the desire for personal causation allows one to act, instead of reacting. This, however, does not mean ruling out externally oriented motivation, but the balance might swing more toward the internally oriented end. As shown in this study, productivity-oriented motivation also made valuable contribution to students' academic performance.

The regression analyses gave us a clearer idea on how the two types of motivation influence students' mathematics achievement collaboratively. Though the two motivational factors together only account for around $10 \%$ of variance in students' final scores, they in most cases demonstrated statistically significant impacts. In particular, students with higher level of pleasure-oriented motivation tended to have significantly better achievement in mathematics in all the nine education systems. The data showed that students from East Asian systems generally received greater benefits from a higher level of PLM than their Western counterparts. In this aspect, students from Chinese Taipei seemed to gain the most achievement increment (i.e. 6.7\%), followed by Korean students (i.e. 5.5\%), and less than half of the potential increment was found in the England sample (i.e. 2.4\%). 
It is found that the impact of productivity-oriented motivation on students' achievement is weaker than that from pleasure-oriented motivation when both types of motivation are taken into account. The greatest influence was observed in Hong Kong followed by Korea; students from the two systems could receive nearly $2 \%$ increment in their achievement with one level higher of PRM. For Singapore and all the four Western samples, a negative impact was found, with the corresponding magnitude in Australia, The Netherlands, and the USA being statistically significant. With this finding, it is not a surprise that educators in the West advocate more of the role of internally oriented motivation in students' learning but deemphasize that of externally oriented motivation. In contrast, while appreciating the importance of intrinsic motivations, educators in East Asia also highly promoted extrinsic motivation. Leung (2001) suggested that such a difference could be related to the fact that people from the East and West had different views on the nature of human beings. According to Leung, East Asians believe that human beings need some "push" in learning, and therefore, providing an optimal level of pressure could well direct students' energy and attention to study. In contrast, Westerners see human beings in a more positive way; they believe that it is enough to arouse students' interest for them to be initiated to learn.

Such differences in cultural belief are also reflected in other aspects in mathematics education. For instance, researchers found that there was an obvious difference in the choice of content in textbooks between East Asia and the West. In particular, students in East Asia are expected to follow the same curriculum and to learn the same content, whereas a variety of curricula are often available to cater for students' different interests and needs in the West. Park \& Leung (2006) argued that it somehow reflected a deeper philosophical difference between the two cultures: "social orientation" versus "individual orientation". That is, the East Asian culture believes in orthodoxy but in the Western culture the individual is of paramount importance. Under the influence of "social orientation" philosophy, students in East Asia are further typically expected to put in hard work and perseverance in learning so as to achieve high scores in examinations as well as bring honors to their family (Hwang, cited in Leu $\& \mathrm{Wu}$ 2006). Teachers there are in turn expected to attain competence in mathematics in order to become experts or models in the field (Park \& Leung 2006). Cai's (2006) US-China comparison found that the difference in cultural values also appeared to affect teachers' scoring practices, that is, while the US teachers tended to give students full credit as long as students can solve problems using whatever viable strategies available, the Chinese 
teachers chose to deduct points for less desirable solutions or written formats in order to help students form good habits.

In fact, the influence of culture on learning and motivation has been recognized by many education and psychology researchers in recent years (Salili \& Hoosain, 2007). As Hernandez \& Iyengar (2001) proposed, people are motivated only when the contexts fostered a perception consistent with their culturally related values or beliefs. Therefore, it is important to take the effects of cultural values, norms, and practices into account when applying operation and effects of academic motivation to students from different cultures (Ng, 2003; Trueba, 1988). Otherwise, we may be misguided by studies solely based on Western theories or concepts and motivate students in East Asia in a culturally inappropriate manner and vice versa. From the current study, it appears that while a higher level of productivity-oriented motivation demonstrates a detrimental effect on the Western students' mathematics learning, utilizing both internally oriented (e.g. pleasure-oriented) and externally oriented (e.g. productivity-oriented) motivation in promoting students' learning is a more effective way for East Asian students than only focusing on either type of motivation.

Lastly, the present analysis was conducted at the system level, and it would be interesting to further investigate the similarities and differences within individual systems, though this is beyond the scope of the current study. Different types of motivation may work differently for students with different achievement level, gender, inclination, etc. within the same system. Through both cross-system and within-system analyses, the influences of different types of motivation on student achievement, as well as the intricate relation between those influences and the underlying cultural values, will be made clear. This will help dispel any simplistic conclusions about the influence of student motivation on achievement.

\section{ACKNOWLEDGEMENT}

The Hong Kong component of TIMSS 2003 was supported by a grant from the Hong Kong Research Grants Council (HKRGC). However, any views expressed in this paper are those of the authors and may not necessarily represent the views of the HKRGC.

Open Access This article is distributed under the terms of the Creative Commons Attribution Noncommercial License which permits any noncommercial use, distribution, and reproduction in any medium, provided the original author(s) and source are credited. 
APPENDIX

List of Motivation Items Related to Mathematics in TIMSS 2003

The following seven questions regarding mathematics were on a fourpoint Likert scale of (1) agree a lot, (2) agree a little, (3) disagree a little, and (4) disagree a lot:

1. I would like to take more mathematics in school (SQ2 8b) BSBMTMOR.

2. I enjoy learning mathematics (SQ2_8d)-BSBMTENJ.

3. I think learning mathematics will help me in my daily life (SQ2_9a) BSBMAHDL.

4. I need mathematics to learn other school subjects (SQ2_9b) BSBMAOSS.

5. I need to do well in mathematics to get into the university of my choice (SQ2 9c)-BSBMAUNI.

6. I would like a job that involved using mathematics (SQ2 9d) BSBMAJOB.

7. I need to do well in mathematics to get the job I want (SQ2_9e)BSBMAGET.

\section{NOTES}

\footnotetext{
${ }^{1}$ Principal component analysis (PCA) is the oldest and most widely used multivariate approach, and the basic idea of the approach is to present the variation of a set of multivariate data in terms of a set of uncorrelated latent factors (Wang 2009), which therefore provides a good remedy for the multicollinearity problem in regression analysis (Malau-Aduli, Aziz, Kojima, Niibayashi, Oshima \& Komatsu, 2004). Correspondingly, one application of PCA is as a preparatory step for regression analysis (Daley, 2008).

${ }^{2}$ Due to the fact that a clear factor structure only appears when BSBMAJOB and BSBMAGET are separated in the analysis, the composite score for each factor was created by averaging measured variables (i.e. giving equal weight to each indicator). Moreover, according to Pedhazur and Schmelkin (1991), adding or averaging the ratings is probably the most frequent approach taken in forming composite scores.
}

\section{REFERENCES}

Amabile, T. M. (1983). The social psychology of creativity. New York: Springer.

Amabile, T. M., Schatzel, E. A., Moneta, G. B. \& Kramer, S. J. (2004). Leader behaviors and the work environment for creativity: Perceived leader support. The Leadership Quarterly, 15, 5-32. 
Baker, L., Dreher, M. J. \& Guthrie, J. T. (Eds.). (2000). Engaging young readers: Promoting achievement and motivation. New York: The Guilford Press.

Bateman, T. S. \& Crant, J. M. (2003). Revisiting intrinsic and extrinsic motivation. Paper presented at the Academy of Management annual meeting, Seattle, WA.

Bolt, M. \& Myers, D. G. (1984). The human connection: How people change people. Downers Grove, IL: InterVarsity Press.

Brophy, J. E. (2004). Motivating students to learn. Mahwah, NJ: Lawrence Earlbaum Associates.

Broussard, S. C. (2002). The relationship between classroom motivation and academic achievement in first and third graders. Unpublished master's thesis, Louisiana State University.

Cai, J. (2006). U.S. and Chinese teachers' cultural values of representations in mathematics education. In F. K. S. Leung, K.-D. Graf \& F. J. Lopez-Real (Eds.), Mathematics education in different cultural traditions: A comparative study of East Asia and the West (pp. 465-481). New York: Springer.

Cameron, J. (2001). Negative effects of reward on intrinsic motivation - a limited phenomenon: Comment on Deci, Koestner, and Ryan. Review of Educational Research, $71(1), 29-42$.

Cohen, J. (2005). AM statistical software (version 0.06.03 Beta). Washington, DC: American Institute for Research. Retrieved from http://am.air.org.

Daley, C. M. (2008). Application of data mining tools for exploring data: Yarn quality case study. Unpublished master thesis. Raleigh, NC: North Carolina State University.

Deci, E. L. (1980). The psychology of self-determination. Lexington, MA: Health.

Deci, E. L. \& Ryan, R. M. (1985). Intrinsic motivation and self-determination in human behavior. New York: Plenum Press.

Deci, E. L. \& Ryan, R. M. (1991). A motivational approach to self: Integration in personality. In R. A. Dienstbier (Ed.), Nebraska symposium on motivation: Perspectives on motivation (Vol. 38, pp. 237-288). Lincoln, NE: University of Nebraska.

Deci, E. L. \& Ryan, R. M. (2000). Intrinsic and extrinsic motivations: Classic definitions and new directions. Contemporary Educational Psychology, 25, 54-67.

Demmrich, A. (2005). Improving reading comprehension by enhancing metacognitive competencies: An evaluation of the reciprocal teaching method. Unpublished doctoral dissertation, University of Potsdam.

Early, S. (2006). Motivational load in instructional design. In G. Clarebout \& J. Elen (Eds.), Avoiding simplicity, confronting complexity: Advances in studying and designing (computer-based) powerful learning environment (pp. 97-108). Rotterdam, Netherlands: Sense.

Evertson, C. M. \& Weinstein, C. S. (Eds.). (2006). Handbook of classroom management: Research, practice, and contemporary issue. Mahwah, NJ: Lawrence Erlbaum Associates.

Foy, P. \& Joncas, M. (2004). TIMSS 2003 sampling design. In M. O. Martin, I. V. S. Mullis \& S. J. Chrostowski (Eds.), TIMSS 2003 technical report. TIMSS \& PIRLS international study center (pp. 108-123). Chestnut Hill, MA: Boston College.

Franken, R. (1994). Human motivation. Pacific Grove, CA: Brooks/Cole.

Geen, R. G. (1995). Human motivation: A social psychological approach. Belmont, CA: Wadsworth.

Gonzalez, E. J., Galia, J. \& Li, I. (2004). Scaling methods and procedures for the TIMSS 2003 mathematics and science scales. In M. O. Martin, I. V. S. Mullis \& S. J. 
Chrostowski (Eds.), TIMSS 2003 technical report. TIMSS \& PIRLS international study center (pp. 253-273). Chestnut Hill, MA: Boston College.

Guilloteaux, M. J. \& Dörnyei, Z. (2008). Motivating language learners: A classroomoriented investigation of the effects of motivational strategies on student motivation. TESOL Quarterly, 42(1), 55-77.

Hannula, M. S. (2006). Motivation in mathematics: Goals reflected in emotions. Educational Studies in Mathematics, 63, 165-178.

Harlen, W. \& Deakin, C. R. (2002). A systematic review of the impact of summative assessment and tests on students' motivation for learning (EPPI-Centre Review). In Research evidence in education library, 1. London: Institute of Education.

Hayamizu, T. (2002). Between intrinsic and extrinsic motivation: Examination of reasons for academic study based on the theory of internalization. Japanese Psychological Research, 39(2), 98-108.

Hebb, D. O. (1955). Drives and the C.N.S. (conceptual nervous system). Psychological Review, 62, 243-254.

Hernandez, M. \& Iyengar, S. S. (2001). What drives whom? A cultural perspective on human agency. Social Cognition, 19, 269-294.

Holden, G. (1990). Motivating tutors. Paper presented at the annual conference of the Association of European Correspondence Schools, Glasgow. Retrieved on November 1, 2008, from http://nettskolen.nki.no/forskning/14/motivate.htm.

Kleinginna, P. R., Jr. \& Kleinginna, A. M. (1981). A categorized list of motivation definitions, with a suggestion for a consensual definition. Motivation and Emotion, 5(3), 263-291.

Kohn, A. (1993). Published by rewards: The trouble with gold stars, incentive plans, A's, praise, and other brides. New York: Houghton Mifflin.

Lepper, M. R., Corpus, J. H. \& Lyengar, S. S. (2005). Intrinsic and extrinsic motivational orientations in the classroom: Age differences and academic correlates. Journal of Educational Psychology, 97(2), 184-196.

Lepper, M. \& Henderlong, J. (2000). Turning "play" into "work" and "work" into "play"; 25 years of research on intrinsic versus extrinsic motivation. In C. Sansone \& J. Harachiewicz (Eds.), Intrinsic and extrinsic motivation: The search for optimal motivation and performance (pp. 257-307). London: Academic Press.

Lepper, M. R., Keavney, M. \& Drake, M. (1996). Intrinsic motivation and extrinsic rewards: A commentary on Cameron and Pierce's meta-analysis. Review of Educational Research, 66, 5-32.

Leu, Y.-C. \& Wu, C.-J. (2006). The origins of pupils' awareness of teachers' mathematics pedagogical values: Confucianism and Buddhism-driven. In F. K. S. Leung, K.-D. Graf \& F. J. Lopez-Real (Eds.), Mathematics education in different cultural traditions: A comparative study of East Asia and the West (pp. 139-152). New York: Springer.

Leung, F. K. S. (2001). In search of an East Asian identity in mathematics education. Educational Studies in Mathematics, 47(1), 35-51.

Li, J., Davis, H. \& Lomax, R. (2008, October). Utilizing factor analysis to examine high school students' academic motivation. Paper presented at the annual meeting of the MWERA, Columbus, Ohio.

Li, P. \& Pan, G. (2009). The relationship between motivation and achievement: A survey of the study motivation of English majors in Qingdao Agricultural University. English Language Teaching, 2(1), 123-128. 
Lim, C. \& Yeon, E. (2009). Review of current studies in instructional design theory in Korea: Major trends and future directions. Asia Pacific Education Review, 10(3), 357364.

Lindenberg, S. (2001). Intrinsic motivation in a new light. Kyklos, 54, 317-342.

Lindenberg, S. (2003). The cognitive side of governance. Research in the Sociology of Organization, 20, 47-76.

Macnab, D. S. \& Payne, F. (2003). Beliefs, attitudes and practices in mathematics teaching: Perceptions of Scottish primary school student teachers. Journal of Education for Teaching, 29(1), 55-68.

Malau-Aduli, A. E. O., Aziz, M. A., Kojima, T., Niibayashi, T., Oshima, K. \& Komatsu, M. (2004). Fixing collinearity instability using principal component and ridge regression analyses in the relationship between body measurements and body weight in Japanese black cattle. Journal of Animal and Veterinary Advances, 3(12), 856-863.

Mankin, K. R., Boone, K. M., Flores, S. \& Willyard, M. R. (2004). What agriculture students say motivates them to learn. NACTA Journal, 48(4), 6-11.

Markus, H. R. \& Kitayama, S. (1991). Culture and the self: Implications for cognition, emotion, and motivation. Psychological Review, 98, 224-253.

Means, T., Jonassen, D. \& Dwyer, F. (1997). Enhancing relevance: Embedded ARCS strategies vs. purpose. Educational Technology Research and Development, 45(1), 517.

Model, E. D. (2005). Creation and validation of the dual motivation profile scale. Unpublished doctoral dissertation, University of Florida.

Ng, C.-H. (2003). Re-conceptualizing achievement goals from a cultural perspective. Paper presented at joint conference of NZARE \& AARE, Auckland, New Zealand.

Ngaosuvan, L. (2004). Motivation and episodic memory performance. Unpublished doctoral dissertation, Umeå Universitet.

Park, K. \& Leung, K. S. F. (2006). A comparative study of the mathematics textbooks of China, England, Japan, Korea, and the United States. In F. K. S. Leung, K.-D. Graf \& F. J. Lopez-Real (Eds.), Mathematics education in different cultural traditions: A comparative study of East Asia and the West (pp. 227-238). New York: Springer.

Pedhazur, E. J. \& Schmelkin, L. P. (1991). Measurement, design, and analysis: An integrated approach. Hillsdale, NJ: Erlbaum.

Rose, H. (2007). Jump-starting student motivation to use self-access learning facilities: A case-study of a class's use of a free-conversation area. The Kanda Journal, 19, 1-18.

Ryan, R. M. \& Connell, J. P. (1989). Perceived locus of causality and internationalization: Examining reasons for acting in two domains. Journal of Personality and Social Psychology, 57, 749-761.

Ryan, R. M. \& Deci, E. L. (1999). Intrinsic and extrinsic motivations: Classic definitions and new directions. Contemporary Educational Psychology, 25, 54-67.

Salili, F. \& Hoosain, R. (Eds.). (2007). Culture, motivation, and learning: A multicultural perspective. Charlotte, NC: Information Age.

Simms, B. (1998). The effect of an educational computer game on motivation to learn basic musical skills: A qualitative study. In S. D. Lipscomb (Ed.), Proceedings of the fifth international technological directions in music education conference (pp. 7-8). San Antonio, TX: International Mountain Society.

Sternberg, R. J. (Ed.). (1999). Handbook of creativity. Cambridge, UK: Cambridge University Press. 
Sternberg, R. J. \& Wagner, R. K. (1994). Mind in context: Interactionist perspectives on human intelligence. New York: Cambridge University Press.

Tang, L. (2008). Beyond intrinsic and extrinsic motivation: Internalized extrinsic motivation in the promotion of learning among primary school students. Paper presented at the annual meeting of the International Communication Association, New York City, NY.

Trueba, H. T. (1988). Culturally based explanations of minority students' academic achievement. Anthropology and Education Quarterly, 19, 270-287.

Vallerand, R. J., Pelletier, L. G., Blais, M. R., Briere, N. M., Senecal, C. \& Vallieres, E. E. (1992). The academic motivation scale: A measure of intrinsic, extrinsic and amotivation in education. Educational and Psychological Measurement, 52, 10031017.

Walker, R. A. \& Debus, R. L. (2002). Educational psychology: Advances in learning, cognition and motivation. Change: Transformations in Education, 5(1), 1-25.

Walker, R. T. \& Guzdial, M. (1999). Collaborative music to motivate mathematics learning. Retrieved October 15, 2008 from http://guzdial.cc.gatech.edu/csc199/CSCLjukebox.pdf

Wang, J. (2008). Stimulating students' motivation in foreign language teaching. US-China Foreign Language, 6(1), 30-34.

Wang, Y. (2009). Statistical techniques for network security: Modern statistically-based intrusion detection and protection. Hershey, PA: Information Science Reference.

Weiner, B. (1990). History of motivational research in education. Journal of Educational Psychology, 82(4), 616-622.

Wittink, D. R. (1998). The application of regression analysis. Needham Heights, MA: Allyn and Bacon.

Wright, B. E. (2004). The role of work context in work motivation: A public sector application of goal and social cognitive theories. Journal of Public Administration Research and Theory, 14(1), 59-78.

Faculty of Education

The University of Hong Kong

Pokfulam, Hong Kong SAR, China

E-mail:yanzhu@hkucc.hku.hk 